\title{
Optical properties of single and double (111)-grown (Ga, In)As-GaAs strained-layer quantum wells under strong photo-injection
}

\author{
P. BORING, K.J. MOORE*, P. BIGENWALD, B. GIL and K. WOODBRIDGE** \\ Groupe d'Etudes des Semiconducteurs, Université de Montpellier II, case courrier 074, 34095 Montpellier \\ cedex 5, France \\ * The Manchester Metropolitan University, Department of Mathematics and Physics, John Dalton \\ Building, Chester Street, Manchester M1 SGD, U.K. \\ ${ }^{* *}$ University College London, Department of Electronic and Electrical Engineering, London WC1E 7JE, \\ U.K.
}

We show that manybody-effects and bandgap renormalization can be easily produced in strained-layer quantum wells with internal built-in piezo-electric fields, under photo excitation. Our observation was made at low temperature by comparing the behaviour of Ga0.92 In 0.08 As-GaAs strained layer single and double quantum wells grown along the (001) and (111) directions when the densities of photoinjected carriers is tuned over several decades. Comparison between experimental data and the results of a Hartree calculation including the space charge effects reveals that manybody interactions are efficiently photo-induced in the (111)-grown samples. Moreover, in the case of double quantum wells, additional transitions appear in the photoluminescence spectra, due to the tunnelling of the two first excited heavy-hole levels for moderate densities.

Semiconductor heterostructures based on III-V and II-VI compounds exhibit excitonic properties which depend on the growth orientation, due to the anisotropy of the valence band of the bulk semiconductors. Strained-layer compounds grown away from the (001) orientation exhibit additional properties connected to the existence of strong built-in piezo-electric fields. The implication of the existence of such piezo-electric fields on conduction and valence states has been investigated in detail by Smith and Mailhiot in a series of pioneering studies on superlattices ${ }^{1}$. Experimental observation of the effect appeared later in the literature for superlattices, single and double quantum wells for a wide range of combinations 2 . The potential of strained layer heterostructures having built-in piezo-electric fields for making self-optic modulators has stimulated us to compare the optical properties of (001)- and (111)B-grown (Ga,In)As-GaAs single and double quantum wells embedded in pin structures. The pin field was set at $1.510^{4} \mathrm{volt} / \mathrm{cm}$ and opposes the piezoelectric field. Figure 1 displays the firs! electron and heavy-hole envelope functions calculated for both kind of structures, for a well width of $10 \mathrm{~nm}$ well, and 10 $\%$ indium. For the (111) growth, the pin field is one order of magnitude smaller than 
the piezo-electric field. The envelope functions of the carriers are strongly spatially separated by the piezo-electric field.

We have calculated the excitonic interaction in such samples in the context of the variational method, using two different trial functions. Model 1 uses a trial function $\Psi_{1}=\chi_{\theta}\left(z_{\theta}\right) \chi_{h}\left(z_{h}\right) \exp (-\rho / \lambda)$, while model 2 uses a more sophisticated trial function: $\Psi_{2}=\chi_{\theta}\left(z_{\theta}\right) \chi_{h}\left(z_{h}\right) \exp \left(-\sqrt{\rho^{2}+\left(z_{e}-z_{h}\right)^{2}} / \lambda\right)$, where $z_{e}, z_{h}, \rho$ are the electron, heavy-hole, and in-plane coordinates respectively. The variational parameter is $\lambda$. Figure 2 (lower part) summarises the results of the variational calculation for heavy-hole exciton. The oscillator strengths have also been calculated and are given in the upper part of figure 2. The reduction of the excitonic interaction in (111)B-grown wide wells, compared with (001)-grown ones is in straightforward relation with the strong spatial separation of the carriers under the effect of the internal piezo-electric field. Model 2 gives higher values for the excitonic interaction, due to the z-contribution to the exciton trial function. Although we compute rather comparable values for the exciton energies, the oscillator strengths display completely different behaviour with well-width. (111)B-grown wide wells should exhibit strong non linear optical properties, due to the existence of the internal piezoelectric field. Thus, we expect large values of the radiative lifetime (it is roughly speaking inversely proportional to the oscillator strength) and subsequent strong space charge effects for (111)B grown samples under laser illumination.

Figure 1

Ga.9 $\ln _{0.1}$ As-GaAs strained-layer quantum wells in pin diodes

$E_{\text {pin }}=1.510^{4} \mathrm{Volt} / \mathrm{cm}$
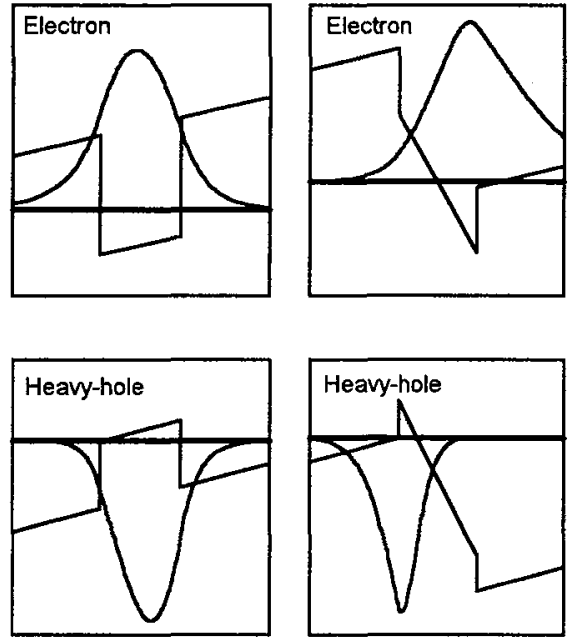

(001)-grown

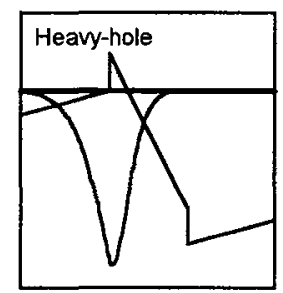

(111)B-grown

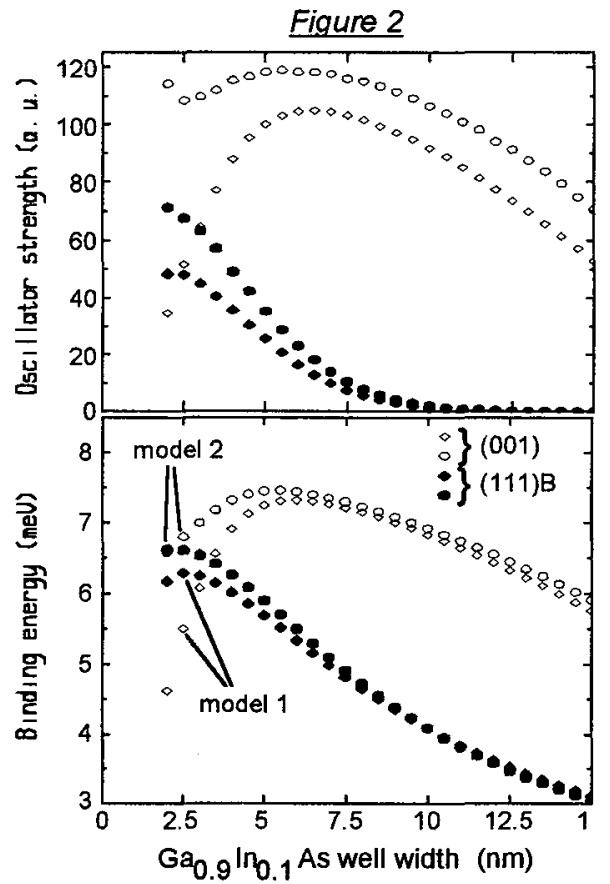

Photoluminescence data taken on both (001)-grown and (111)B-grown samples have shown completely different behaviour; the photoluminescence energy doesn't significantly vary for the (001)-grown sample, whilst, for the (111)B-grown sample it exhibits a linear dependence with pump power at moderate power 
densities and saturates under high excitation conditions. This blue-shift of some 25 $\mathrm{meV}$ has also been observed in double quantum wells grown along the (111) direction.
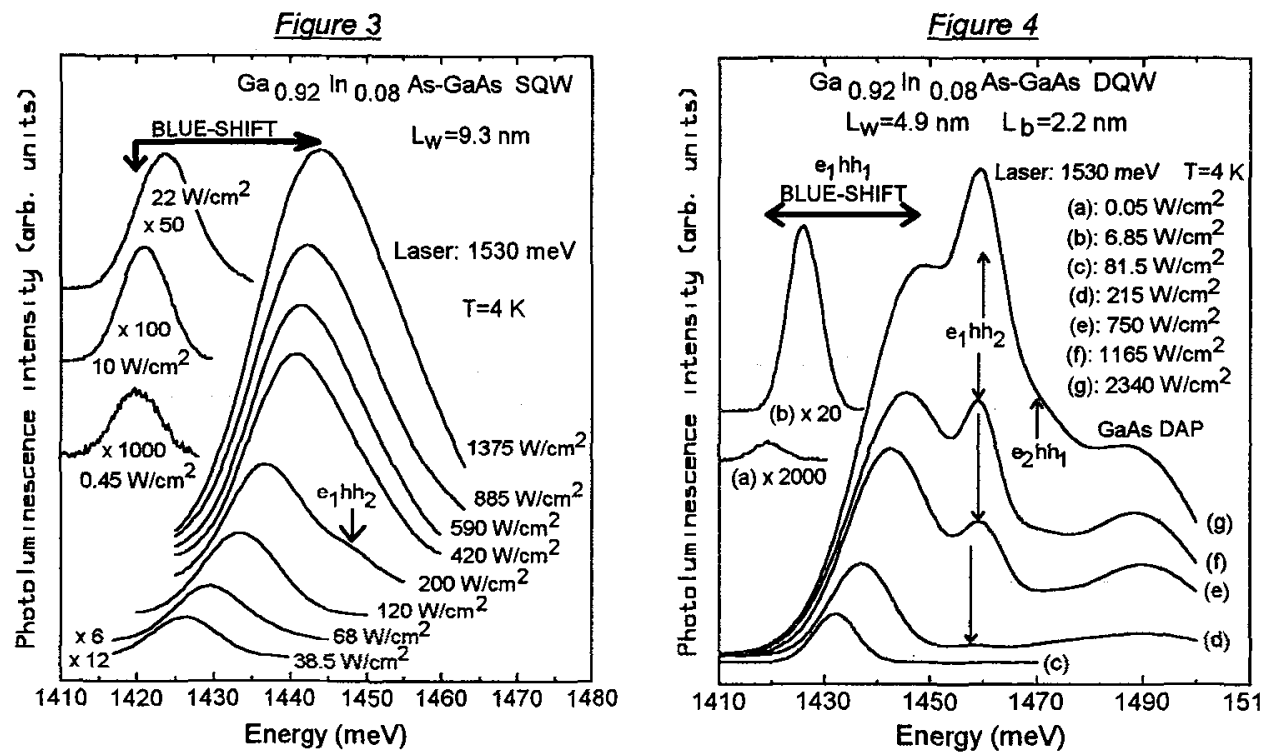

Figures 3 and 4 summarise the evolution of the photoluminescence signal for two (111)B-grown structures: a single and a double quantum well. We note that the main photoluminescence line broadens and that additional lines appear at high injection density. This we attribute to phase space filling by photo carriers. The observation of the additional features is linked to the crossing of the hole (electron) Fermi energy with the second heavy-hole (electron) subband at sufficient photocarrier densities. To explain our observations we must consider a number of phenomena. Firstly, the effect of space charge fields caused by the spatial separation of photo-injected electrons and holes. To calculate the magnitude of the space charge effect, and its implications on the PL with pump power, in the simplest approach, we have to solve self-consistently the equation:

$$
\left[-\frac{\hbar^{2}}{2} \frac{\partial}{\partial z}\left(\frac{1}{m_{j}^{*}(z)}\right) \frac{\partial}{\partial z}+V(z)+q[F(z)+\Phi(z)] z\right] \chi(z)=E \chi(z)
$$

for each type of carrier. In this equation, $V$ is the potential line-up, $F$ is the total field without injected carriers, and $\Phi$ is the contribution of photo-injected electron-hole pairs. In the most general way, at T OK, $\Phi$ is defined as:

$\Phi(z+d z)-\Phi(z)=e \sigma \int_{z}^{z+d z}\left[\sum_{m, l}^{k_{\perp}(\sigma)} \int_{0}^{\alpha_{m, i}}\left(k_{\perp}, \sigma\right) \chi_{h_{m, i}}^{2}\left(k_{\perp}, u\right) d k_{\perp}-\sum_{n, j} \int_{0}^{k_{\perp}(\sigma)} \beta_{n, l}\left(k_{1}, \sigma\right) \chi_{e_{n, j}^{2}}^{2}\left(k_{\perp}, u\right) d k_{\perp}\right] \frac{d u}{\varepsilon(u)}$ 
where the summations $\Sigma$ are extended over the $n$ electron (e) and $m$ hole $(h)$ states of interest. Given an areal carrier density $\sigma$, one will fill one or several confined subbands up to an appropriate value of the in plane wave vector $k_{\perp}$. The indices $i$ and $j$ refer to the lifting of spin degeneracy away from $k_{\perp}=0$, and $\varepsilon(z)$ is the dielectric constant.

Figure 5 displays the result of the calculation for the samples of figures 3 and 4. The area of the circles is proportional to the oscillator strength between conduction and valence envelope functions. Our calculation accounts for the experimental data (bending and saturation of the photoluminescence energy), but overestimates the blue-shift. This is attributed to the onset of manyboby interactions and band gap renormalisation (BGR) at high carrier densities. The values we estimate for this quantity, for the single quantum well are plotted on figure 6 as the differences between the calculated and the measured energy (open diamonds). Our data reasonably matches with results of three theoretical calculations (crosses, full and open circles) for GaAs-(Al, Ga)As.
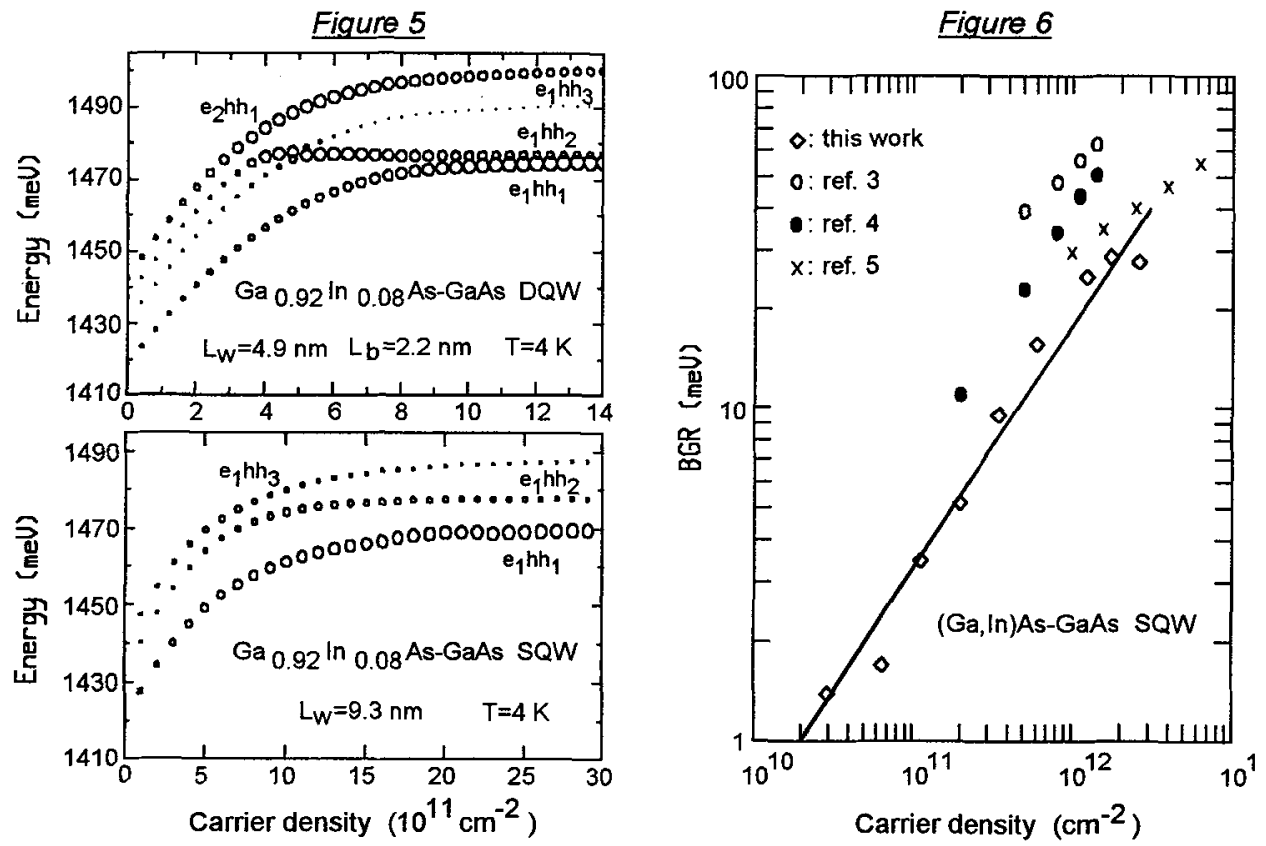

\section{References}

1 - D.L.Smith and C.Mailhiot, Reviews of Modern Physics, 62, 173, (1990)

2 - P.Boring, B.Gil, and K.J.Moore, Phys. Rev. Lett., 71, 1875, (1993) and refs. therein

3 - S.Das Sarma, R.Jalabert, and S.R.Eric-Yang, Phys. Rev B, 41, 8288, (1990)

4 - P.Von Allmen, Phys. Rev B, 46, 13 345, (1992)

5 - J.C.Ryan, and T.L.Reinecke, Phys. Rev, B, 47, 9613, (1993) 